PREPARED FOR THE U.S. DEPARTMENT OF ENERGY, UNDER CONTRACT DE-AC02-76CH03073

PPPL-3570

PPPL-3570

UC-70

\title{
Magnetic Probe Construction Using Thick-Film Technology
}

H. Takahashi, S. Sakakibara, Y. Kubota, and H. Yamada

May 2001

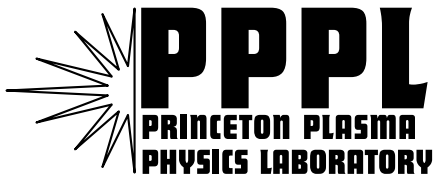

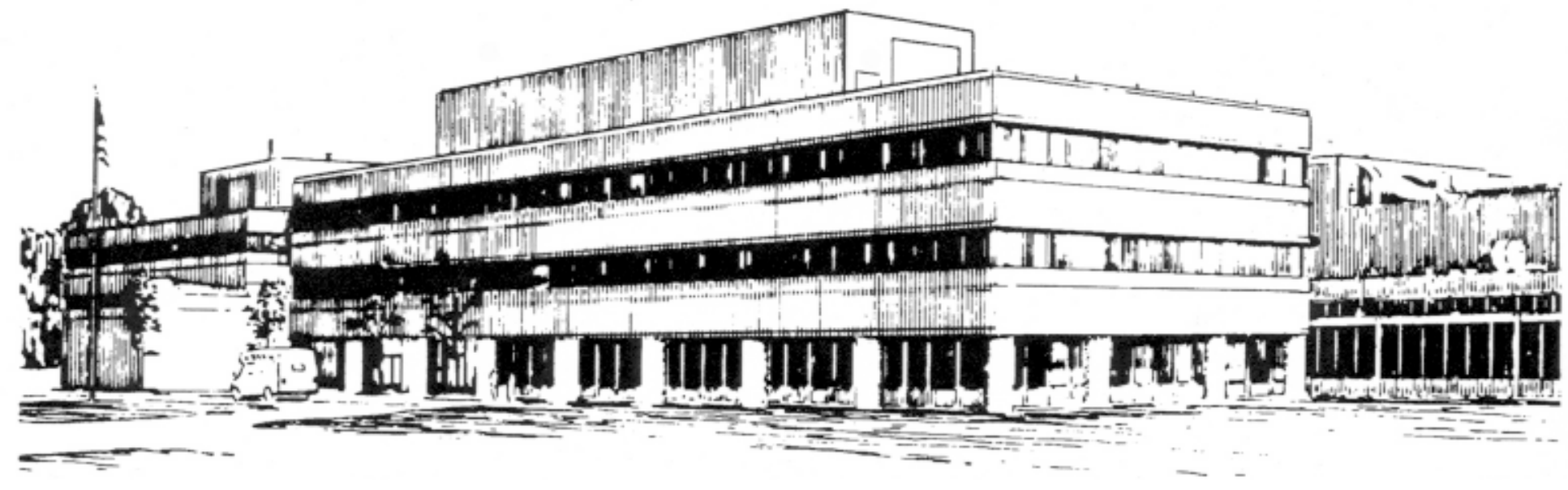

PRINCETON PLASMA PHYSICS LABORATORY PRINCETON UNIVERSITY, PRINCETON, NEW JERSEY 


\section{PPPL Reports Disclaimer}

This report was prepared as an account of work sponsored by an agency of the United States Government. Neither the United States Government nor any agency thereof, nor any of their employees, makes any warranty, express or implied, or assumes any legal liability or responsibility for the accuracy, completeness, or usefulness of any information, apparatus, product, or process disclosed, or represents that its use would not infringe privately owned rights. Reference herein to any specific commercial product, process, or service by trade name, trademark, manufacturer, or otherwise, does not necessarily constitute or imply its endorsement, recommendation, or favoring by the United States Government or any agency thereof. The views and opinions of authors expressed herein do not necessarily state or reflect those of the United States Government or any agency thereof.

\section{Availability}

This report is posted on the U.S. Department of Energy's Princeton Plasma Physics Laboratory Publications and Reports web site in Calendar Year 2001. The home page for PPPL Reports and Publications is: http://www.pppl.gov/pub_report/

DOE and DOE Contractors can obtain copies of this report from:

U.S. Department of Energy

Office of Scientific and Technical Information

DOE Technical Information Services (DTIS)

P.O. Box 62

Oak Ridge, TN 37831

Telephone: (865) 576-8401

Fax: (865) 576-5728

Email: reports@adonis.osti.gov

This report is available to the general public from:

National Technical Information Service

U.S. Department of Commerce

5285 Port Royal Road

Springfield, VA 22161

Telephone: 1-800-553-6847 or

(703) 605-6000

Fax: (703) 321-8547

Internet: http://www.ntis.gov/ordering.htm 


\title{
MAGNETIC PROBE CONSTRUCTION USING THICK-FILM TECHNOLOGY
}

\author{
H. Takahashi \\ Princeton Plasma Physics Laboratory, Princeton University \\ Princeton, New Jersey, U.S.A. \\ and \\ S. Sakakibara, Y. Kubota, H. Yamada \\ National Institute for Fusion Science \\ Toki-shi, Gifu-ken, Japan
}

\begin{abstract}
Thick-film technology has been successfully adapted for the design and fabrication of magnetic probes of a new type suitable for use in the simultaneous ultra-high vacuum and high temperature environment of a nuclear fusion device. The maximum usable temperature is expected to be around $900 \mathrm{deg} \mathrm{C}$. This new probe has a specific sensitivity (coupling area per unit volume) an order of magnitude higher than a conventional coil. The new probe in one implementation is capable of simultaneously measuring magnetic field in three orthogonal directions about a single spatial point and in two frequency ranges. Low-frequency coils have a measured coupling area of 296-323 $\mathrm{cm}^{2}$, and a frequency response of about $300 \mathrm{kHz}$. High-frequency coils have a design coupling area of $12-15 \mathrm{~cm}^{2}$.
\end{abstract}

\section{INTRODUCTION}

Magnetic coils for measuring magnetic field are a widely used diagnostic and sensing tool in many fields, including scientific research, industrial, and commercial applications. The development of magnetic coils reported in this article is an outgrowth of work conducted in nuclear fusion and plasma physics devices, in particular, tokamaks and stellarators, which one day are expected to evolve to electricity-generating nuclear fusion reactors. But the instrument described here should find applications, not only in other plasma-related research fields, but also in industrial and commercial arenas.

Nuclear fusion and plasma physics research investigates behavior of a plasma trapped and heated in a magnetic configuration. The plasma often responds to trapping and heating by generating its own usually oscillating magnetic field known as a Magneto-Hydro-Dynamic (MHD) instability. Magnetic coils are an indispensable instrument ${ }^{1}$ for studying the nature of a magnetic trap itself and MHD oscillations generated in it.

A universal requirement for coils used in these applications is their compatibility with an ultrahigh vacuum environment. The use of such coils ${ }^{2}$ in a nuclear fusion reactor would add a new dimension to their design that is much harder to satisfy: the survivability under intense thermal and nuclear radiation. Magnetic probes must be able to withstand a much harsher environment with an essentially zero failure rate.

Yet, unlike magnetic sensing devices in industrial and commercial applications, the design technology of magnetic coils used in fusion and plasma research fields has remained essentially 
unchanged for many decades. A magnetic coil used in these fields is essentially a length of wire wound on a bobbin. Aside from some variations in the type of wire used, for example, mineralinsulated wire (MI) or co-axial cable, the construction of a coil remained the same. Evidence for inactivity in this development area is the paucity of formal literature ${ }^{3-6}$ to cite.

Material and technology exist today, in the form of printed circuits on ceramic substrates, for fabricating magnetic probes that are more compact yet more rugged than the conventional wound wire types. The construction advantages refer to mechanical, thermal, electrical, vacuum environmental, and possibly also nuclear radiation resistant properties of the instrument.

The existence of the necessary technology, however, is only in principle. Practical issues abound that require resolution through actual proto-typing and testing. Prominent among them are the choice of material and fabrication method: adhesion of metallic ink to substrate and delamination during fabrication, substrate machinability, accuracy of the internal structure with respect to external dimensions, damage under thermal cycling and shocks, radiation damage, vacuum compatibility, etc.

This article is exclusively about magnetic coils - a type of magnetic sensing device based on the law of induction for detecting time varying field. But the instrument described here is actually a set of such coils with an electrostatic shield around them, all in a monolithic body. The word magnetic probe will designate a whole device in this article. These new probes will be referred to as AT probes, consistent with the 'Advanced Technology' applied to them.

The AT probes can contribute to fusion research in present day facilities, not only as a possibly more reliable replacement for existing probes, but also in opening new frontiers that few conventional probes have explored. For example, highly compact and rugged probes can be placed much closer to the plasma with a minimum of protection, and new areas of physics study such as measurement of short (down to a few millimeters) wavelength magnetic fluctuations at the plasma edge become available. The ability of these probes to withstand a high temperature environment is indispensable in a steady-state device in which removing accumulated heat from every probe may be impractical or costly. Such probes are also valuable in a pulsed device with first walls at very high temperatures.

\section{DESIGN FEATURES}

The design of AT probes evolved through several stages. Early models were all single-axis coils, which used planar spirals as a coupling element. They developed into the latest tri-axial model, which employed both planar spirals and helices as coupling elements. We designed and built early models to test feasibility of various technical options. We will discuss primarily the latest model in this article.

An AT probe of the latest design measures three orthogonal components of magnetic field in two frequency ranges. The targeted low frequency range is up to several hundred $\mathrm{kHz}$, and the targeted high frequency range is up to a few tens of $\mathrm{MHz}$. It measures all three field components with a sensitivity (coupling area in $\mathrm{cm}^{2}$ ) comparable to coils used in today's fusion research, and has an electrostatic shield. It has a rectangular shape. One design was optimized for use in the Large Helical Device ${ }^{7}$ (LHD) - a superconducting stellarator at the National Institute for Fusion Study in Japan. The design features of this Model-LHD probe are further elaborated below. 
A prominent characteristic of the AT probes is their compact size. Figure 1 compares a coil of a conventional design ${ }^{8}$ (background) used in the TFTR tokamak, ${ }^{9}$ two early single-axis probes (foreground left and middle) called Model-K and Model-N, and a latest tri-axial two-frequency probe (foreground right), Model-LHD. Table I compares physical dimensions and coupling areas of some of these probes. The Model- $\mathrm{K}$ is similar in characteristics as the Model-N other than some technical options employed for the internal construction.

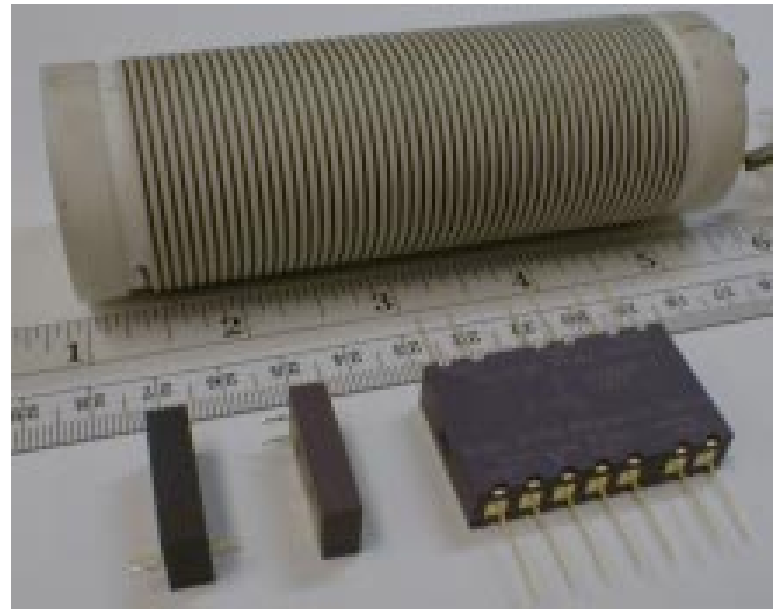

Fig. 1 A conventional coil (background) and Advanced Technology (AT) probes (foreground).

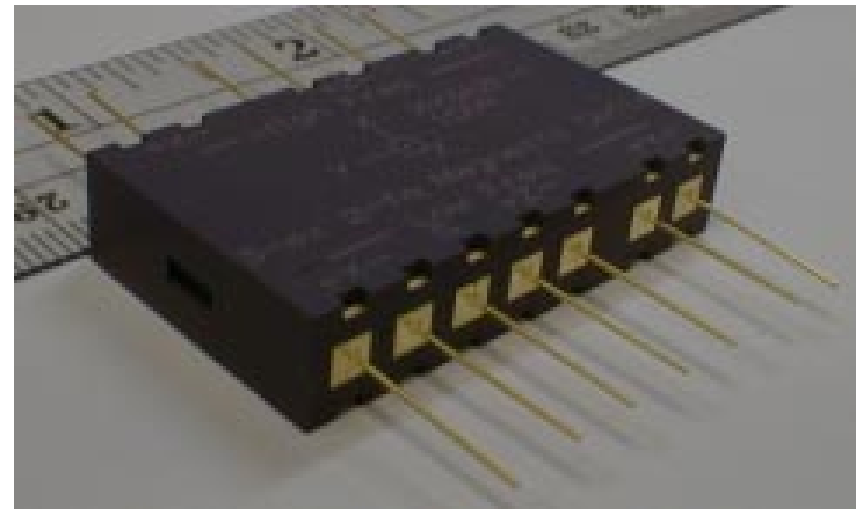

Fig. 2 A Model-LHD AT probe contains six sensing elements - for three orthogonal directions in low and high frequency ranges - and an electrostatic shield.

A parameter, 'specific sensitivity,' may be useful in making such a comparison. It is a coil's coupling area divided by its volume. The parameter is also listed in the table for these coils. The single-axis Model-N probe, with a spiral coupling element, achieved a highest $182 \mathrm{~cm}^{-1}$ specific sensitivity. The tri-axial Model-LHD probe attained $124 \mathrm{~cm}^{-1}$. The coupling area here is the sum of all coils within a probe. The TFTR coil reached $4.8 \mathrm{~cm}^{-1}$. Either model of AT probes has a specific sensitivity that is an order of magnitude greater than that of a TFTR coil. The latter design was, however, not specifically optimized on the basis of compactness.

Table I: Parameters of Advanced Technology (AT) and Conventional Coils.

\begin{tabular}{|r|c|c|c|}
\hline & Conventional & \multicolumn{2}{|c|}{ Advanced Technology } \\
\hline & TFTR & Model-LHD & Model-N \\
\hline Dimensions $(\mathrm{X} / \mathrm{Y} / \mathrm{Z})(\mathrm{cm})$ & $3.8 \mathrm{D} / 10 \mathrm{~L}$ & $2.4 / 4.0 / 0.85$ & $1.1 / 2.2 / 0.5$ \\
Volume $\left(\mathrm{cm}^{3}\right)$ & 113 & 8.4 & 1.2 \\
Coupling Area $\left(\mathrm{cm}^{2}\right)$ & 550 & 1045 & 220 \\
Specific Sensitivity $\left(\mathrm{cm}^{-1}\right)$ & 4.8 & 124 & 182 \\
\hline
\end{tabular}

An AT probe has seven main circuit elements and six auxiliary circuit elements. Main circuit elements are three low frequency coupling coils, three high frequency coupling coils, and an electrostatic shield. Three coupling coils of each frequency range couple to magnetic field components in three orthogonal directions. For example, an X-axis coil couples a field component along the $\mathrm{X}$-axis. The electrostatic shield reduces the extraneous probe response caused by electrostatic coupling to the probe's surrounding. Six auxiliary circuit elements are simple 'pass through' 
conducting paths that serve as signal return 'wires' in a series connection of two or more probes.

A magnetic coil in general measures magnetic field averaged over a volume in space. Six coils in an AT probe average field over volumes of different shapes and sizes. But all volumes are centered about a single shared point in space (volumetric center of the entire probe). The probe thus measures three orthogonal components of field about a single point in space in two frequency ranges. We call it a tri-axial two-frequency probe.

A coupling element is either a multi-layered series-connected set of helices, each of a rectangular cross-sectional shape, or a stack of series-connected planar spirals. Each helical coupling coil consists of two substantially identical series-connected helices that are displaced along their common axis symmetrically from the coil (probe) center. Each spiral coil consists of two seriesconnected subsets of planar spirals that are displaced along their common axis symmetrically from the coil (probe) center. These design features assure that the volume within every coil is centered about the probe center.

The electrostatic shield is a 'bird-cage-like' structure that lets through magnetic field, but provides a substantially contiguous surface of equal electrostatic potential. The 'roof' and 'floor' of the bird cage are contiguous conducting surfaces except for slits cut into them to break up induced current eddies into smaller sizes.

Table II: Design Parameters of Coupling Elements in AT Probe

\begin{tabular}{|r|c|c|c|c|c|c|}
\hline & \multicolumn{3}{|c|}{ Low Frequency } & \multicolumn{3}{c|}{ High Frequency } \\
\hline & X-coil & Y-coil & Z-coil & X-coil & Y-coil & Z-coil \\
\hline No. of layers & 8 & 8 & 18 & 8 & 8 & 2 \\
No. of turns & 227 & 268 & 252 & 6 & 12 & 4 \\
Trace Length $(m)$ & 16.64 & 13.53 & 8.43 & 0.55 & 0.66 & 0.33 \\
Coupling Area $\left(\mathrm{cm}^{2}\right)$ & 315.1 & 328.8 & 290.9 & 11.70 & 14.99 & 13.07 \\
\hline
\end{tabular}

The main means of external electrical connection is through gold-plated terminal pins. But the probe also provides recessed flat surfaces which a contact point can be pressed against or can be brazed on. These flat surfaces, together with 'pass through' auxiliary circuits, also serve as a means of series-connecting any number of probes for attaining a greater sensitivity. The probe has provisions for installation without placing holding clamps over the probe 'top' surface.

Table II lists some of these design parameters.

\section{DESIGN IMPLEMENTATION}

The thick-film technology implements the circuit design of an AT probe. It is a well established manufacturing process used for making, for example, a base board for a computer chip. But the sheer number of layers and complexity of patterns in the present application still pushes back its technical frontiers and stretches manufacturing experience.

The circuit element is a contiguous structure of electrically conducting material embedded in a body of insulating ceramic material. The manufacturing process begins with a stack of soft 'green ceramic' sheets. A screening procedure prints circuit patterns on usually a 'top' face of each sheet 
with electrically conducting metallic ink. This ink is a mixture of particulates of a refractory metal (often, tungsten, sometimes, molybdenum and others) and ceramic powder suspended in a volatile solvent. Ceramic powder promotes ink adhesion. Holes punched through a sheet and filled with ink interconnect patterns on adjacent faces. Such a hole is often called a 'via.' A kiln 'co-fires,' or 'fires simultaneously,' green ceramic and metallic ink in the stack at temperatures ranging up to $1500 \operatorname{deg} C$, after it is pressed together and given provision for escaping gases. Heat drives out most volatile agents from green ceramic and ink. The stack turns into a monolithic body of hard ceramic with electrically conducting residues of metallic ink embedded in it. An AT magnetic probe of the present design uses 40 sheets, each about $0.2 \mathrm{~mm}$ thick (post-firing dimension). A stack of standard four-inch-square sheets contains several probes that are cut out after co-firing, using a saw.

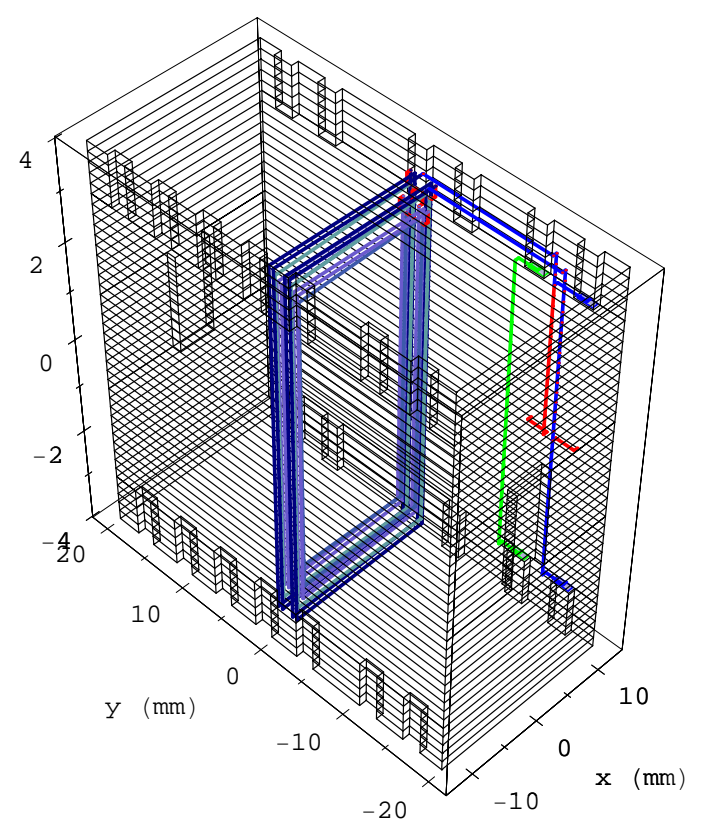

Fig. 3 An 'x-ray view' of a high frequency Y-axis coil. A stack of green ceramic sheets is shown, each by a black outline with its thickness greatly exaggerated. Lines in blue are the coil body proper. Lines in red are coil leads. Lines in green are an auxiliary circuit.

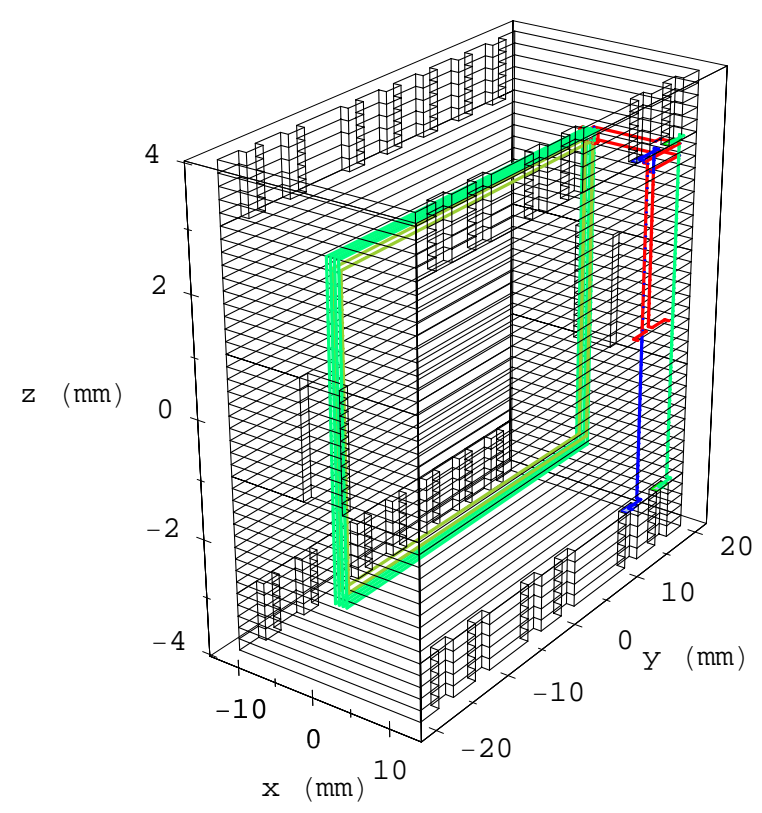

Fig. 4 An 'x-ray view' of a high frequency X-axis coil. It fits through the opening of the $\mathrm{Y}$-axis coil shown in the previous figure.

We illustrate by examples how circuit elements are constructed within a ceramic body. Figure 3 is an 'x-ray view' of a Y-axis coil. A stack of green ceramic sheets is shown, each by an outline with its thickness greatly exaggerated. Lines in blue are the coil body proper. Individual turns are not well distinguishable in this scale. But a 'horizontal rung' of a turn is a line printed on the face of a sheet, and a 'vertical leg' is a series of vias punched through a stack of sheets. The winding advances in pitch along the helix axis only on a horizontal run. A vertical leg runs perpendicular to the axis without advancing in pitch. The coil body consists of two helices that are set off symmetrically from the probe center. Lines in red show parts of leads, and lines in green are a return 'wire'. Figure 4 is an X-axis coil. It too consists of two helices that are set off symmetrically from the probe center (but are not distinguishable in this scale). It is at a right angle to, and fits through the opening of, the Y-axis coil. These are high frequency coils. Each has only a few layers, and each layer has only a few turns. See Table II. A low frequency coil has more layers 
and a much larger number of turns in each layer, and is not suitable for illustrative purposes: it fills a large fraction of the probe volume, and obscures other elements.

A Z-axis coil is a set of planar spirals. Its winding spirals inward on a sheet, interconnects to the next sheet through a via, and then spirals outward. Any even number of such pairs of spirals can form a Z-axis coil. A Z-axis coil consists also of two subsets of spiral pairs that are set off symmetrically from the probe center.

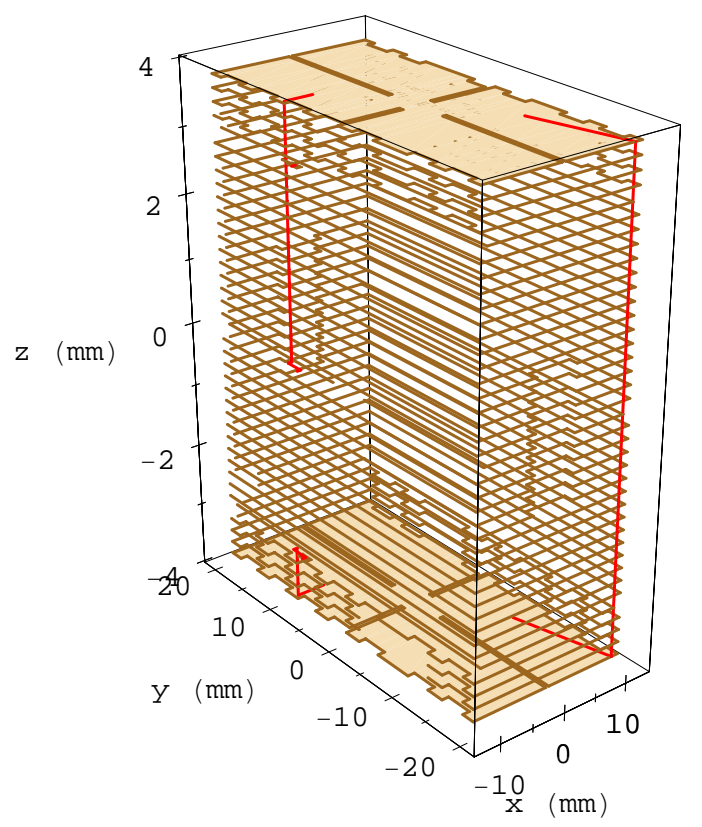

Fig. 5 An 'x-ray view' of an electrostatic shield. Its elements are electrically connected together at a single point in each of them.

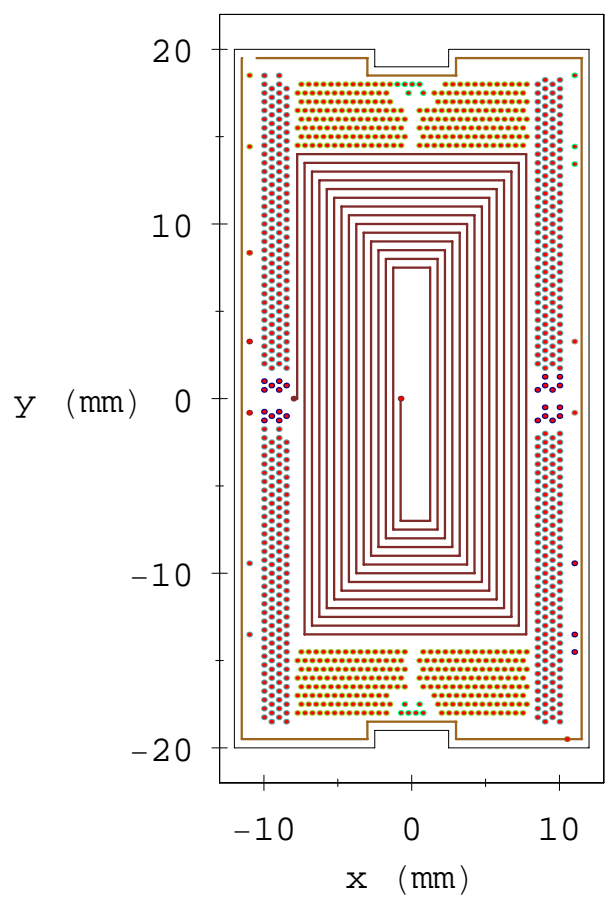

Fig. 6 A metallic ink pattern of a typical plate (22nd sheet out of 40 counting from the bottom). It shows most prominently a spiral element of a Z-axis coil. It also shows cross sections of vertical legs of other coils, leads, and circuit elements.

The electrostatic shield is in Fig. 5. An edge line circumnavigates the periphery of each sheet. It does not close onto itself, however. The gap prevents current from being driven around it. Sheets at the very top and bottom of a probe have their top faces essentially completely covered with metallic ink. These ink sheet patterns have slits cut into them to shorten field penetration time. All ink sheet patterns and edge lines are connected together at a single point in each of them. The interconnection is a red line at a corner of the cage in the figure. A terminal for connecting the shield system to an outside circuit is also shown as a red line.

A sheet (or more precisely its top face) occupies the middle 'height' of a probe $(\mathrm{Z}=0)$. It contains no circuit elements on its face other than inter-connecting leads. On either side of it are spirals composing a high frequency Z-axis coil. Sheets containing a low frequency Z-axis coil come next. X-axis coils, both low and high frequency, wrap around the core. Y-axis coils, both low and high frequency, go over the enlarged core. Finally, the electrostatic shield cage surrounds everything. 
A metallization pattern for each ceramic sheet specifies the actual design of a probe. An example is shown in Fig. 6. It is the 22nd sheet (out of 40) counting from the bottom. It shows most prominently a spiral element of a Z-axis coil. It also shows cross sections of vertical legs of other coils, leads, and circuit elements. Lines represent the surface to be metallized, and 'dots' represent vias. Color coding is for presentation only, and distinguishes coils to which metallized elements belong.

Each circuit consists of joined straight line segments whether running horizontally or vertically. Each joint is a 'node' defined by coordinates in a three-dimensional space. The present AT probe design contains over thirty thousand such nodes. This presents a unique challenge. Moving a few nodes, for example, in a design optimization process, often sets off migration of hundreds or even thousands of nodes. The problem is not unlike those encountered in designing a multiple component geometrical optics device. Only much worse. Lines and vias are packed essentially everywhere within the probe volume as close to each other as manufacturing tolerance allows. Moving a large number of nodes creates a high risk of having some of them violating rules of placing lines and vias. Lines may cross each other in an unintended manner (internal 'shorts'). Or a circuit may no longer be a continuous path ('open circuit') after changes. Vias and lines may come closer than manufacturing tolerance. Updating drawings every time changes occur is necessary to avoid these problems. Even highly sophisticated CAD (Computer Aided Design) devices may not be capable of automatically propagating such massive changes.

We have dealt with some of these issues by programing node coordinates in a Mathematica ${ }^{10}$ program. We represent a circuit as a linear array of elements, each being a set of three coordinates of a node, and take advantage of Mathematica's ability to sort and extract elements according to their characteristics. For example, the program can cull all elements having an identical Zcoordinate and put them on the same sheet. It recognizes and sorts 'planar nodes' and 'via nodes.' It has also been used to draw many figures in this article.

\section{FABRICATION}

We explain some external features of the probe using its overview photograph in Fig. 2. The $\mathrm{X}$-axis is along a shorter side of the probe, the $\mathrm{Y}$-axis is along the longest side, and the Z-axis is perpendicular to the probe face. See Table I for external dimensions. A right-handed coordinate system, engraved on the probe face, shows a positive direction of each axis. The X and Y external dimensions are accurately controllable as individual probes are diced out of a large block by a diamond saw. The Z-dimension is more difficult to control accurately because of contraction of the green ceramic upon firing. But with some experimentation it should be possible to control it within a small fraction of a millimeter. All probe faces are ground to $50 \mu \mathrm{m}$ flatness. Terminal pin designations for low- and high-frequency coils, a coil I.D. (serial number), and a coordinate system are laser engraved on the probe surface as they must survive repeated exposure to a high temperature environment.

Electrical connection to the low frequency coils and the electrostatic shield is through seven terminal pins on a long side of the probe: a pair of pins each for the X-, Y-, and Z-axis coils, and a single pin for the shield. Connection to the high frequency coils is through six pins on an opposite side: a pair each for the $\mathrm{X}-, \mathrm{Y}-$, and Z-axis coils. Each terminal pin is $0.45 \mathrm{~mm}$ in diameter and $12.7 \mathrm{~mm}$ in length, and is made of 'Kovar' (Fe-Ni-Co alloy). It is brazed to a metallized 
patch on a side of the probe body with a silver-based braze material having a melting point not lower than $920 \mathrm{deg}$ C. The pins and the patches are gold plated. For each coordinate direction a positive voltage $V$ appears on a terminal designated as '+' with respect to its '-' counterpart, when magnetic field $B$ aligned with a positive axis of that coordinate decreases with time in accordance with the law of induction, $V=-A d B / d t$, where $A$ is the coupling area.

The probe body has a notch cut directly above and below each terminal pin. Its 'bottom' surface is metallized and gold plated. Seven top notches are visible in the figure above the low frequency coil terminal pins. Each coil has two pairs of notches associated with it. Two members of a pair are electrically paralleled with the coil's terminal pins. Two members of the other pair are electrically connected to each other inside the probe body by a return 'wire.' The shield has only a pair of notches associated with it. They are connected to the shield's terminal. All of these notches and their metallized surfaces serve double purposes. First, they are an alternative means of effecting electrical connection when a terminal pin is not usable or convenient. An electrical contact can be pressed against a metallized surface, or connected by other means, e.g., brazing or electrically conducting adhesive. Second, the notches and their metallized surfaces serve as a means of joining more than one probe in series when the coupling area of a single coil does not meet a requirement. When two coils are stacked, one on top of another, adjacent notches form a 'well,' with their metallized surfaces facing each other. Filling wells with electrically conducting material will accomplish a series connection of the two coils. Any number of coils can in principle be stacked in this manner. A last coil of a stack needs a jumper wire between one end of each coil to its adjacent return 'wire' in a multi-probe configuration.

The probe body has yet another pair of indentations or 'dimples' on its shorter sides. One of them is visible in the figure as a dark rectangle. They are for mounting purposes. Space in one direction is at a premium in many applications; for example, a probe must stay within the shadow of a limiter in a fusion device. This is in fact the reason why the present design has a z-dimension much smaller than the other two dimensions. Placing a holding mechanism over a probe body would use up that precious room. In other applications a metallic holding structure over a probe body may shield out high frequency field. These 'dimples' on the sides of a probe can accommodate a clamping mechanism in such situations.

\section{TESTING}

\section{A. Structural Tests}

Co-firing of a stack of green ceramic substrates causes it to contract, and introduces uncertainty in the dimensions as well as the internal orientation of sensing elements within the resultant monolithic ceramic body. The dimensional uncertainty affects the probe sensitivity. The orientation matters when aligning the axis of a sensing element using a probe's exterior surface as a reference. The magnitude and statistical variations of the uncertainty are a subject of magnetic testing described in a later section.

The exterior dimensions of a probe in an X-Y plane are accurately machined as an 'area' of specified dimensions containing sensing elements is sawed out of a block of ceramic. But this dimensional accuracy does not necessarily imply that the sensing elements lie accurately 
parallel or perpendicular to a probe's exterior surface, because the locations of registration marks (holes) provided on green ceramic sheets have also changed upon co-firing. Uneven changes in the registration marks would result in an internal orientation inaccuracy.

Contraction of green ceramic is less predictable in an out-of-plane direction (along Z-axis) than in in-plane directions. The overall thickness of AT probes from the first production batch came out to be $8.7 \mathrm{~mm}$, about $9 \%$ greater than the design value. The excess thickness resulted in greater coupling areas of the X-axis and Y-axis coils. Missing the targeted thickness in the first attempt was in most part due to the lack of experience with a large number of layers involved. The metallic ink volume also amounted to $1.6 \%$ of the ceramic volume because of a high density of circuits - a factor that was not adequately taken into account. We will refer to these probes as 'first batch' probes.

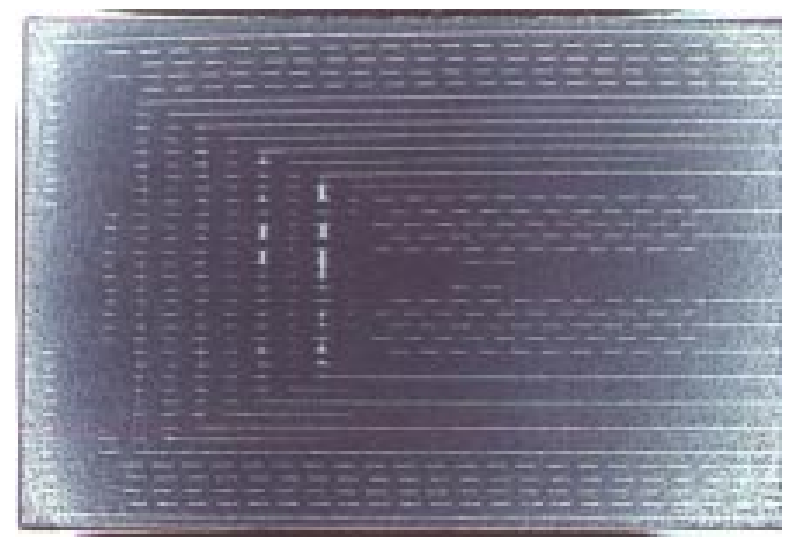

Fig. 7 Parts of a cross section (Y-Z plane) of an AT probe after co-firing. Horizontal 'lines' and 'dashes' are metallization seen in cross section. Thicker vertical 'lines' are vias seen in cross section.

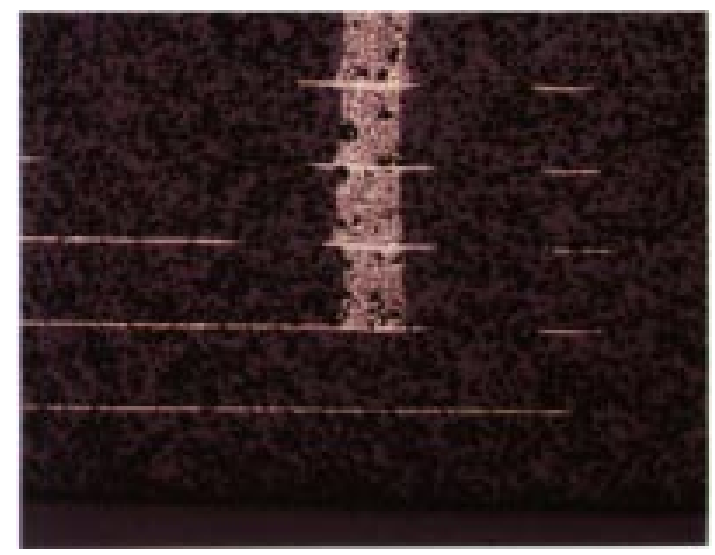

Fig. 8 An enlarged cross section of an AT probe, showing a column of vias. They are formed well and in contact with their neighboring members.

In an attempt to reduce the probe thickness, later batches of probes were sent to a different manufacturing plant, which uses a somewhat different co-firing procedure. The overall thickness diminished only slightly to $8.5 \mathrm{~mm}$, about $6 \%$ greater than the design value. But the coupling areas of the $\mathrm{X}$-axis and $\mathrm{Y}$-axis coils became much closer to their design values. We will refer to these probes as 'later batches' probes. We plan to use in the future slightly thinner (non-standard) green ceramic sheets to meet the design thickness.

In order to examine an interior structure a probe body was sliced off in a plane perpendicular to the X-axis. Figure 7 shows parts of a cross section (Y-Z plane). It encompasses the entire probe thickness (Z-direction), but only about a third of a horizontal dimension (Y-direction). Horizontal 'lines' and 'dashes' are metallization seen in cross section. Thicker short vertical 'lines' are vias seen in cross section. The figure demonstrates that a stack of green sheets has formed after cofiring a good monolithic ceramic body without any signs of delamination and other irregularities. Major delamination, which could occur in a large multi-layer structure, would compromise the mechanical integrity of the probe body.

Figure 8 shows an enlarged part of the cross section that includes a column of vias. They are formed well and in contact with their neighboring members. Localized delamination, or an internal void would cause neighboring vias to separate, and produce an open circuit. Figure 9 
shows another enlarged part that illustrate how the internal circuit connects to an external terminal pin. The large white area is a cross section of a brazed terminal pin base. Figure 10 shows a small part of the cross section in a greater magnification. It demonstrates well-formed metallization. Its thickness is about $15 \mu \mathrm{m}$. The width is close to the design value of $300 \mu \mathrm{m}$. The cross-sectional area is about $4.5 \times 10^{-3} \mathrm{~mm}^{2}$.
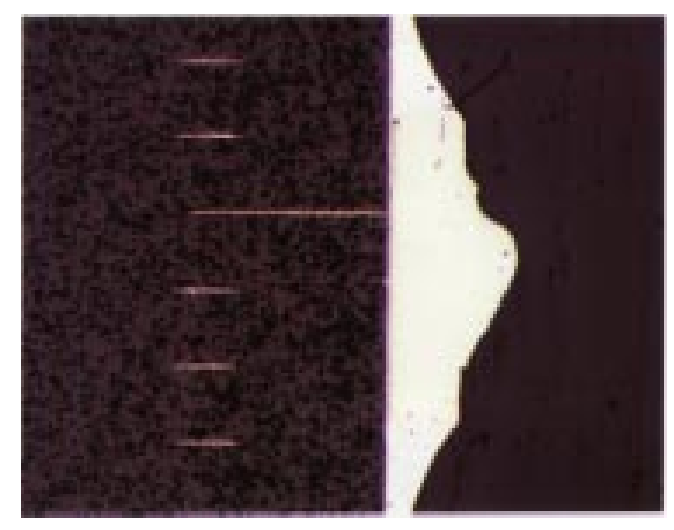

Fig. 9 An enlarged cross section of an AT probe, showing an external connection. A large white area is a cross section of a brazed terminal pin base.

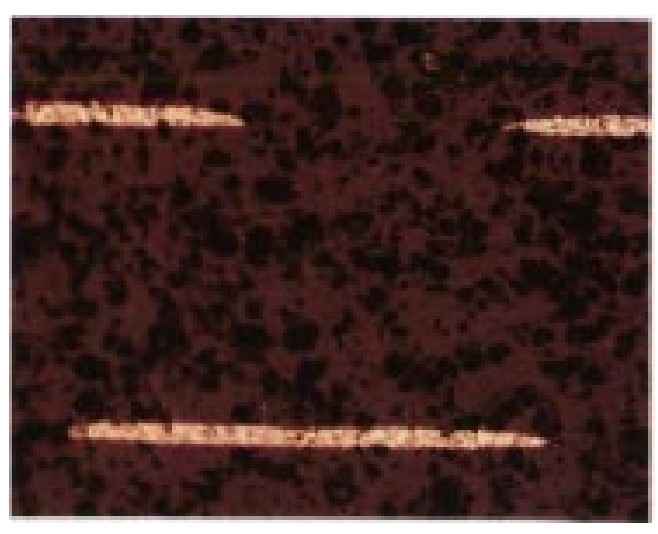

Fig. 10 A greatly enlarged cross section of an AT probe, showing well-formed metallic traces. The trace width is about $300 \mu \mathrm{m}$, and the thickness is about $15 \mu \mathrm{m}$ at the trace center.

\section{B. Thermal and Vacuum Tests}

Thermal cycling and vacuum compatibility tests utilized a Thermal Desorption Spectrometer ${ }^{11}$ (TDS) at NIFS. The TDS measures gases with mass numbers up to 60 .

All the tested samples were the physically smaller Model-N probes built in an earlier phase of the development. Some samples were only a probe body proper, and others were complete probes with terminal pins attached. These early models used silver-based braze material with a melting point of not less than $780 \mathrm{deg} \mathrm{C}$ to attach terminal pins to the body.

An outgassing test heated four probe bodies proper with a total weight of about $19 \mathrm{~g}$ to 1000 deg C. It recorded no outgassing measurable above a background level. Another test heated four complete probes to $700 \mathrm{deg} \mathrm{C}$ and held that temperature for $30 \mathrm{~min}$. It recorded no outgassing measurable above a background level.

A thermal cycling test subjected four probe bodies proper to 102 heating/cooling cycles between $200 \mathrm{deg} \mathrm{C}$ and $700 \mathrm{deg}$ C. Each cycle lasted $25 \mathrm{~min}$. The test produced no obvious mechanical changes to the probe bodies other than discoloration. Sample temperature was brought down to a room temperature three times over the course of the test, and the electrical resistance of internal circuits was measured. It varied by as much as 3\% among the three measurements. But the changes were not secular.

The upper limit of a usable temperature range for the AT probe comes presently from melting of braze material used to attach terminal pins. The latest design probes use silver-based braze material with a melting point not lower than $920 \mathrm{deg}$ C. The probes are usable theoretically up 
to about $900 \operatorname{deg} \mathrm{C}$.

A magnetic probe used in a pulsed fusion device will experience cyclical heating and cooling. The relative emissivity of probe surfaces is a parameter of interest, because the peak temperature attained depends strongly on it. In the absence of conduction cooling, the probe temperature, T, will follow a radiative heat transfer equation,

$$
\frac{M C_{p}}{A} \frac{d T}{d t}=-\epsilon \sigma_{S B} T^{4}+S
$$

where $M, C_{p}, A, \epsilon, \sigma_{S B}$, and $S$ are the mass, specific heat, surface area, relative emissivity, Stefan-Boltzman constant, and a heat source term.

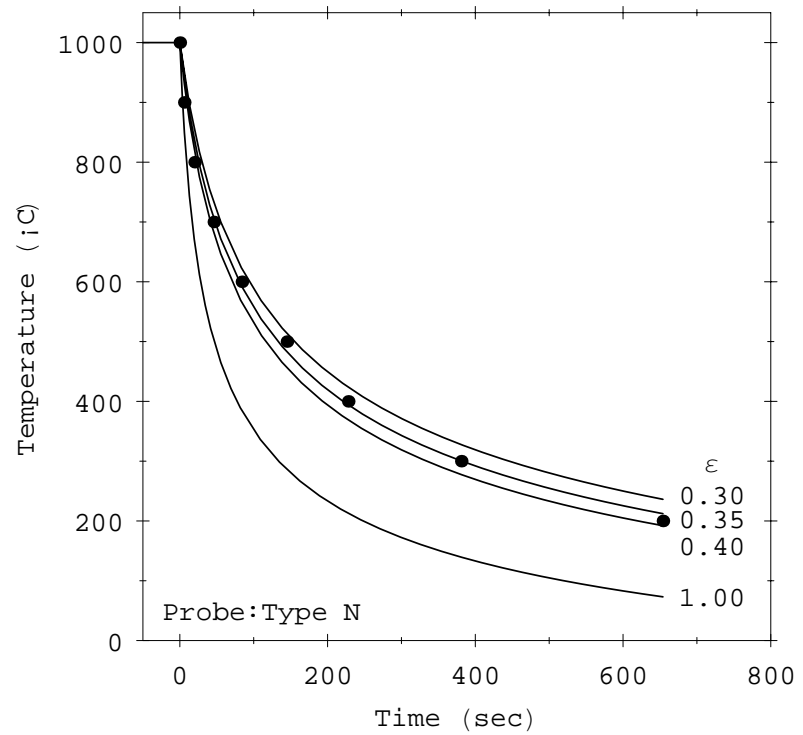

Fig. 11 Emissivity and cooling characteristics of an AT probe body. Curves are solutions to a radiative heat transfer equation (Eq. 1) for several relative emissivity values. 'Dots' are measured values.

Figure 11 shows solutions to the above equation in the absence of a heat source with a $1000 \operatorname{deg} \mathrm{C}$ initial temperature for several relative emissivity values. The $\epsilon=1$ curve is the black body radiation. We do not have information on the specific heat of the probe material, but used a value, $C_{p}=800 \mathrm{~J} / \mathrm{kg} / \operatorname{deg} \mathrm{K}$, quoted for a ceramic of alumina content (96\%), which is higher than the probes (approximately 94\%). Overlaid on these curves are 'dots' indicating temperatures of the sample during a radiation cooling test using a single probe body proper. These are values at discrete time points transcribed from a chart recorder trace. The dots fall generally between $\epsilon=0.35-0.4$. These are perhaps surprisingly low values, considering the dark brown color of the probe surfaces. We will take the $\epsilon=0.4$ value as it appears more appropriate at higher temperatures, and also assume that it applies to all models of AT probes.

\section{Electrical Tests}

Electrical testing consists mainly of continuity checks and resistance measurements. It also verifies the absence of an inter-circuit short. An intra-circuit short is much more difficult to 
detect unless it jumps a large number of turns or layers, and causes the circuit resistance to fall precipitously.

The electrical resistance of a coil in the AT probe is generally large, reaching several hundred ohms, because it is made of a mixture of refractory metal and ceramic particulates. Table III lists for a 'later batches' probe (KY3A2F-1N1050) the measured electrical resistance as well as the specific surface resistivity computed from it. The nominal width of the trace was $300 \mu \mathrm{m}$ in the computation.

Table III: Measured Properties of Coupling Elements in AT Probe

\begin{tabular}{|r|c|c|c|c|c|c|c|}
\hline \multicolumn{2}{|c|}{} & \multicolumn{3}{|c|}{ Low Frequency } & \multicolumn{3}{c|}{ High Frequency } \\
\hline Property & Units & X-coil & Y-coil & Z-coil & X-coil & Y-coil & Z-coil \\
\hline Resistance & $\Omega$ & 513 & 348 & 277 & 16.9 & 18.1 & 10.7 \\
Surface Res. & $m \Omega / \square$ & 9.2 & 7.7 & 9.9 & 9.2 & 8.2 & 9.7 \\
Coupling Area & $\mathrm{cm}^{2}$ & $315.7(0.8)$ & $323.2(0.8)$ & $296.1(0.1)$ & & & \\
Coil Axis & $\mathrm{deg}$ & $-0.46(0.19)$ & $-0.31(0.07)$ & $-0.26(0.07)$ & & & \\
Inductance & $\mu \mathrm{H}$ & 313 & 217 & 252 & 1.2 & 2.5 & 0.8 \\
\hline
\end{tabular}

We have experimented with a few options for reducing the resistance in an early stage of the development. Use of wider metallic trace and larger diameter vias will lead to a lower resistance, but at a cost of a reduced specific sensitivity. Choice of Molybdenum over Tungsten also helps, but it is a less commonly selected metallic ink. Triple screening of metallic ink increased the thickness of $200 \mu \mathrm{m}$-wide trace to about $50 \mu \mathrm{m}$ at the trace center. This produced surface resistivity of about $2.2 \mathrm{~m} \Omega / \square$. This represents a reduction of a factor of $4-5$ from values listed in Table III. But the gain came at a significantly increased risk of delamination.

A magnetic diagnostics system should take this high sensor internal resistance into consideration during its design phase. An amplifier or integrator for an AT probe will need an input impedance higher than values common for conventional copper wire coils in order to reduce signal loading. A high input impedance instrument may in turn be more prone to electrostatic noise. The present AT probe design incorporates an electrostatic shield for this reason.

\section{Goals of Magnetic Tests}

Magnetic tests of AT probes serve both production and end-use purposes. The tests would help in detecting production defects. They would also quantify ranges of variability in the sensitivity and the orientation of the axis of each sensing element, and thus help in assessing the need for individual (rather than sample) calibration of probes.

We cannot easily inspect the internal structure of an AT probe for possible defects. It is a drawback of circuits densely packed in an opaque material. A comparison of observations and theoretical expectations of the coupling sensitivity could reveal internal defects. It is not limited to a simple comparison of numerical values of the sensitivity as a coil lies with its axis aligned with a uniform field. Its variation with the angle between the coil axis and field could tell us something about its internal structure. For example, an internal short in a coil not only reduces its coupling area, but can lead to a change in its topology that manifests itself as a change in the angular variation of the coupling sensitivity. We have not yet performed the required theoretical calculations. We hope to complete this aspect of the magnetic test in the future. 
It depends upon requirements of a specific application whether or not the variability in characteristics necessitates calibration of individual AT probes. In some applications the variability may be small enough to satisfy their requirements by calibrating a few sample probes out of a production batch. In others, uncertainties arising from other sources, such as installation inaccuracies (position and external orientation of the probe body) within a large experimental device, may exceed the variability in the sensitivity and internal orientation of sensing elements within the probe body, in which case sample calibration may again be adequate.

It is outside the scope of this article to quantify requirements of specific applications. But we may give broad guideline values, taking a plasma fusion experiment as an example.

A study of MHD oscillations usually has less stringent requirements, which are met perhaps with a $10 \%$ sensitivity accuracy. But reconstruction of an equilibrium state of a magnetic trap demands an accuracy on the order of $1 \%$ or better. For a high sensitivity accuracy to be meaningful, it must be matched by a comparable instrument position accuracy. The sensitivity and position accuracies are in fact more or less interchangeable, because the scale length of the field inhomogeneity is on the order of the device size.

Accurate installation of magnetic probes in a large experimental device is difficult and costly. With the aid of a sophisticated positioning device and accurately known reference points, it is probably possible to place a magnetic probe with an accuracy of about $1 \mathrm{~mm}$. But this is only a static picture. Under vacuum and electromagnetic forces the vacuum vessel distorts, and moves magnetic probes installed on it. The positional uncertainty becomes much greater under such dynamic conditions. Orientation accuracy in installing a magnetic probe is a few degrees at best. These mechanically attainable positional and orientation accuracies are often inadequate for equilibrium reconstruction in a magnetic trap.

In order to overcome inaccuracies introduced during installation we often have to rely on 'software' corrections. An iterative experiment/theory scheme finds likely 'true' position and orientation of a magnetic probe. Experimental data are obtained through exciting a subset of coils that generates a magnetic trap. The observed values are compared with theoretically expected values for an assumed position and orientation of a probe. Given accurately known sensitivity and internal orientation of a sensing element, the software corrections would reduce to search for solutions in a fewer dimensional parameter space, i.e., only in a position/orientation domain. This is an important justification for pursuing accurate sensitivity calibration of magnetic probes.

The orthogonality between the magnetic axes of sensing elements is sometimes an important issue. The lack of orthogonality could mean that aligning a sensing element properly leads to mis-aligning the others. An ideal magnetic trap has no field component perpendicular to its flux surfaces. A magnetic coil placed with its axis perpendicular to a flux surface can register MHD instability signals when the trap deviates from an ideal state. The proper orientation of a sensing element with respect to the flux surface is crucially important in such applications, because field components in directions tangent to a flux surface can be many orders of magnitude greater than the perturbation field component to be measured. 


\section{E. Helmholtz Coil Facility for Magnetic Tests}

With these goals in mind we have designed and built a Helmholtz coil facility at NIFS to calibrate AT probes. It was designed to generate a $5 \mathrm{~cm}$ cube volume at its center with a field non-uniformity not exceeding $0.1 \%$. Each constituent coil of the Helmholtz coil pair has a $45 \mathrm{~cm}$ average radius, ten 'pancake-type' windings, and six turns in each pancake. The windings are made of magnet wire of a $7 \times 7 \mathrm{~mm}$ cross section with a $4 \times 4 \mathrm{~mm}$ cross section cooling channel. Tap water from the municipal water supply with a head up to $3 \mathrm{~kg} / \mathrm{cm}^{2}$ and a throughput of $20 l /$ min runs through twenty (two coils times ten pancakes per coil) paralleled cooling channels. Two DC power supplies connected in parallel, each capable of delivering $100 \mathrm{~A}$ at $35 \mathrm{~V}$, power the Helmholtz coil, which generates up to 235 G DC at its center. The Helmholtz coil sits on a nonconducting (wooden) pedestal to avoid field distortion by the steel-reinforced concrete floor. The coil axis is $1.9 \mathrm{~m}$ above the floor. A stand-alone data acquisition system ('LabView') digitizes and archives signals from an AT probe and a current monitor for the Helmholtz coil. The digitization rate is $1 \mathrm{kHz}$. The Helmholtz coil was calibrated by a Hall-effect probe traceable to a secondary standard by the manufacturer.

An AT probe is mounted on a turn table with its turning axis in a vertical direction. An 'L-shaped' holder bolted to the table provides vertical and horizontal reference surfaces. The estimated angle setting accuracy of the vernier turning table is $\pm 0.05 \mathrm{deg}$. The table slides on an non-magnetic optical bench placed through the opening of the Helmholtz coil. The bench is aligned with the axis of the Helmholtz coil, using cross hair marks scribed on its structure during its fabrication process and a helium-neon alignment laser. The estimated alignment accuracy is \pm 3 milliradian $( \pm 0.17 \mathrm{deg}$ ) between the the Helmholtz coil's structural axis and the optical bench. The structural axis provides a reference direction for the nominal angle setting of the turn table.

It is the direction of the Helmholtz coil's magnetic axis that is relevant. We determined this direction by utilizing the TFTR Mirnov coil shown in Fig. 1. It has a conventional design, and consists of a cylindrical bobbin made of machinable ceramic and a helical winding as the sensing element. The bobbin has exterior dimensions machined to a tight tolerance. It has also a helical groove cut on its surface to hold the winding in place. As the bobbin and the helical winding share the same axis, aligning of the bobbin using its exterior surface as a reference is substantially the same as aligning the helical sensing element. The coil registered a maximum response when its axis was at a nominal angle of -0.63 deg measured from the Helmholtz coil's structural axis. This angle will be taken as the Helmholtz coil's magnetic axis. A physically meaningful quantity is the angle with respect to this magnetic axis. But we will quote, unless otherwise stated, the nominal angle setting of a probe with respect to the structural axis for operational convenience.

\section{F. Magnetic Tests}

A 'first batch' probe (KY3A2F-1N1001) was set up on the turn table in a 'ZX orientation.' The probe's positive Y-axis was parallel to (but not necessarily coincident with) the table's turning axis, and pointing upward. The 'bottom' face (negative Z-axis side) of the probe body, pressed against a vertical reference surface of the L-shaped holder, was a defining surface for the probe's orientation. An 'end' face (negative Y-axis side), pressed against a horizontal reference surface of the L-shaped holder, was another defining surface for the probe's orientation. In this orientation the Z-axis coil would show a maximum signal when the table is set nominally at $0 \mathrm{deg}$. The 
$\mathrm{X}$-axis coil would show a maximum signal nominally at $+90 \mathrm{deg}$. The Y-axis coil, nominally perpendicular to the field at all angle settings, would be the 'non-coupling coil.'

The Helmholtz coil's current signal is shown in Fig. 12. Signals from three low frequency coils are also shown for a probe angle setting of $0 \mathrm{deg}$. Both $\mathrm{X}$-axis and $\mathrm{Y}$-axis coils are nominally perpendicular to the field at this setting. Their signals are in a hundred-fold expanded scale. Signal values during a 'flat-top' period with respect to those well before the start of the Helmholtz coil current are used in calculating the coupling area. An average of the last ten data points before cutting off the current is taken as the flat-top value. Eddy currents are expected to have decayed sufficiently by this time. The test took measurement at closely spaced angle intervals in vicinities of 'zero crossings' of both Z-axis and X-axis coils.

Figure 13 summarizes the sensitivity measurement. It shows signals from three low frequency coils, integrated numerically once with respect to time, as a function of the angle setting of the turning table. The Y-coil data are shown in a gray shade, and in a hundred-fold expanded scale. 'Dots' are measured points. Curves are a least RMS (Root-Mean-Square) error fit to measured points for the $\mathrm{X}$-axis and Z-axis coils. The fitting function is $A_{s} \sin \theta+A_{c} \cos \theta$, where $\theta$ is the turning table angle, and $A_{s}$ and $A_{c}$ are coefficients to be determined from the fitting. A maximum response angle is found from a peak of a fitted curve.

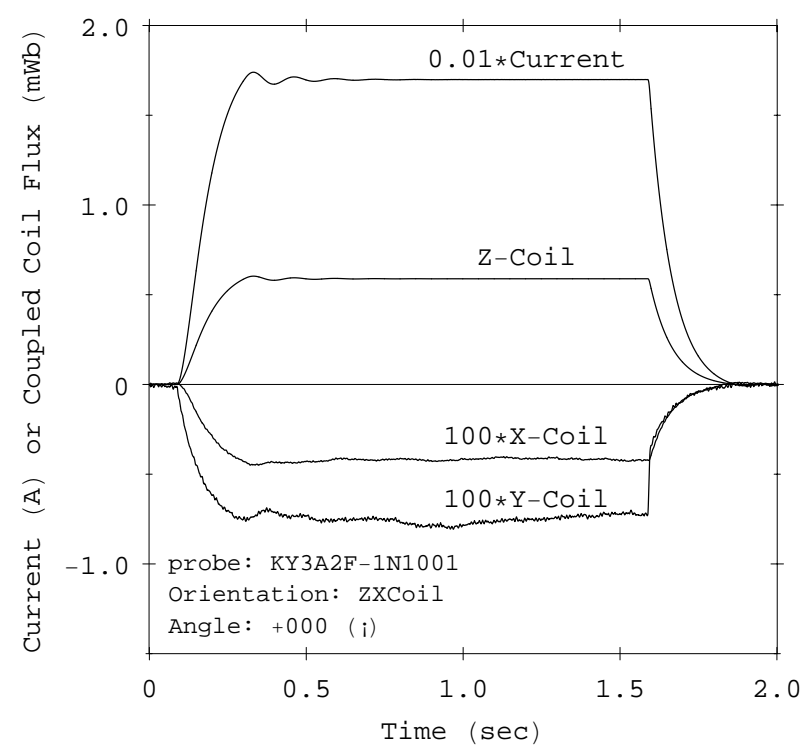

Fig. 12 Signals from low frequency coils in an AT probe placed in a Helmholtz coil with a zero nominal angle between the probe and the Helmholtz coil axis. The $\mathrm{X}$ axis and $\mathrm{Y}$-axis coils are nominally non-coupling coils at this choice of the angle, and their signals are in a hundred-fold expanded scale.

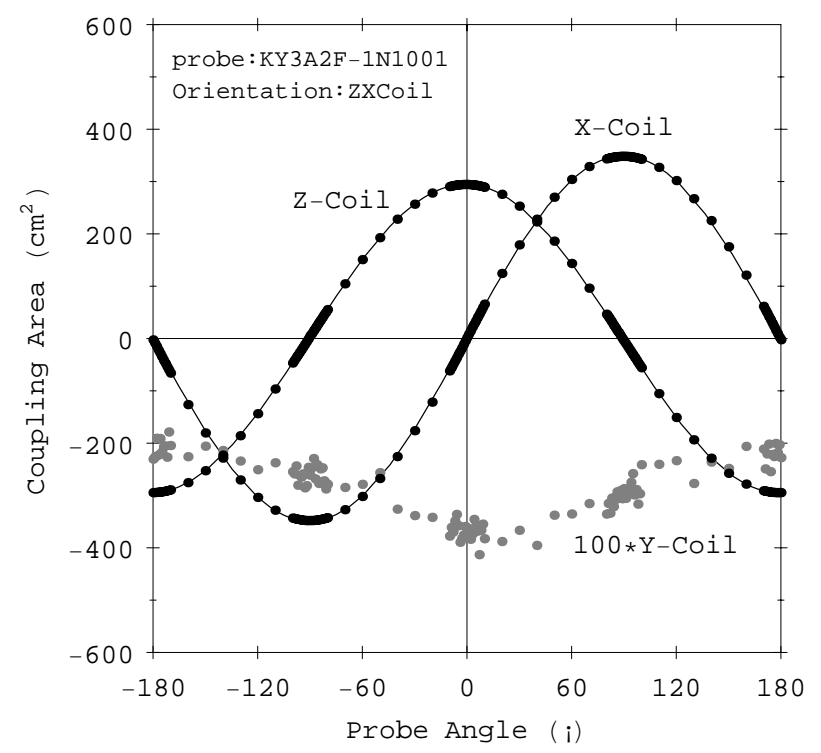

Fig. 13 Coupling areas (sensitivity) of low frequency coils in an AT probe as a function of the nominal angle between the probe and the Helmholtz coil axis. Curves are the least RMS error fit to the Z-axis coil and Xaxis coil signals. The $\mathrm{Y}$-axis coil is nominally a noncoupling coil at all angles. Its coupling area values are on a hundred-fold expanded scale.

A maximum response for the Z-axis coil occurred at a nominal angle of -0.89 deg with a coupling area of $294.6 \mathrm{~cm}^{2}$. The corresponding result was at $+89.64 \mathrm{deg}$ with $348.9 \mathrm{~cm}^{2}$ for the $\mathrm{X}$-axis coil. We will take a maximum response angle, when referred to the magnetic axis, as the direction of an effective axis of a coil. Recall that the Helmholtz coil's magnetic axis is at $-0.63 \mathrm{deg}$. The Z-axis coil and $\mathrm{X}$-axis coil signals peaked at $-0.26 \mathrm{deg}$ and $+0.27 \mathrm{deg}$ away from 
their respective expected directions (magnetic axis direction for the Z-axis coil and 90 deg plus magnetic axis direction for the $\mathrm{X}$-axis coil). The two coil axes made a $90.53 \mathrm{deg}$ angle.

Note that the expected or ideal response is no coupling for the Y-axis coil. It shows instead a 'DC' component of $-2.85 \mathrm{~cm}^{2}$ and a sinusoidally oscillating component of $0.78 \mathrm{~cm}^{2}$ peak amplitude. These numbers are respectively $0.79 \%$ and $0.21 \%$ of the maximum coupling area of the Y-axis coil. A likely source of the oscillating component is a tilt of the rotation axis. When converted to an angle, an extraneous coupling of $0.21 \%$ corresponds to a tilt of $0.12 \mathrm{deg}$. This is within the estimated alignment accuracy. We suspected that the 'DC' component came from extraneous vertical field generated by the Helmholtz coil, but have not verified it independently.

We repeated similar measurements with an 'XY orientation.' The Z-axis coil is the nominally non-coupling coil. For the $\mathrm{Y}$-axis coil the direction of an effective axis was $-1.56 \mathrm{deg}$, and the coupling area was $357.9 \mathrm{~cm}^{2}$. For the $\mathrm{X}$-axis coil the corresponding values were $+89.26 \mathrm{deg}$ and $350.4 \mathrm{~cm}^{2}$. The effective axis was at $-0.93 \mathrm{deg}$ for the $\mathrm{Y}$-axis coil and at $-0.11 \mathrm{deg}$ for the $\mathrm{X}$-axis coil, both measured from their respective expected directions. The two coil axes made a $89.18 \mathrm{deg}$ angle.

The two probe orientations produce two sets of measurements for the $\mathrm{X}$-axis coil, and a set of measurement each for the $\mathrm{Y}$-axis and Z-axis coils. The measured Z-axis coil coupling area came out to be only $1.3 \%$ bigger than the design value. The measured $\mathrm{X}$-axis coil (average of two measured values) and Y-axis coil coupling areas were 11.0 and $8.9 \%$ greater than the design values. Recall that the test sample was a 'first batch' probe.

\section{G. Statistical Variations}

We performed magnetic tests similar to those described in the previous subsection on eleven 'later batches' probes to obtain information on statistical variations of the measured properties. The tests were done only for low frequency coils. Table III summarizes values of the mean and the RMS variance for the coupling area and the angle of the effective axis of sensing elements. The mean of the effective axis angles is measured from the expected maximum response direction.

The coupling areas of the 'later batches' probes differ from the design values by $+0.2,-1.7$, and $+1.8 \%$ for the $\mathrm{X}$-axis, $\mathrm{Y}$-axis, and Z-axis coils, respectively. The RMS variance is $0.22,0.25$, and $0.03 \%$ for the $\mathrm{X}$-axis, Y-axis, and Z-axis coils, respectively. The closeness of the mean of a coupling area to its design value may be somewhat fortuitous except possibly for the Z-axis coil, considering that the probe total thickness differed significantly from its design value. But the smallness of the RMS variance is significant. The deviation of an angle of maximum response from an expected value was $-0.46,-0.31$, and $-0.26 \mathrm{deg}$ for the $\mathrm{X}$-axis, $\mathrm{Y}$-axis, and Z-axis coils, respectively. These values are significantly smaller than installation inaccuracies commonly encountered in nuclear fusion devices. Furthermore, the RMS variance of the angular deviation is only 0.19, 0.07, and $0.07 \mathrm{deg}$ for the $\mathrm{X}$-axis, Y-axis, and Z-axis coils, respectively. The mean value of the angle between the effective axes of the $\mathrm{X}$-axis and Y-axis coils is $90.02 \mathrm{deg}$. The corresponding mean angle for the $\mathrm{X}$-axis and Z-axis coils is $89.66 \mathrm{deg}$.

It thus appears possible to fabricate probes with coupling areas within a few percent of design values through the thick-film technology. The variance within a production batch can be substantially smaller than one percent. It is necessary to measure the sensitivity of only a representative 
number of probes in a batch for most applications. The effective axis of each sensing element can be aligned within about half a degree using a probe's external surface as a reference. The orthogonality of the axes of sensing elements is accurate to within about a third of a degree.

\section{H. Frequency Response}

A quantity of interest in assessing the frequency response of a sensing element is its inductance. We measured the inductance of sensing elements using a Hewlett-Packard Precision LCR Meter, Model 4284A. A sensing element of a 'later batches' probe (KY3A2F-1N1050) was connected to the instrument through four $50 \mathrm{~cm}$ long RG-58 coaxial cables, each with a BNC connector at one end and a pair of alligator clips at the other. The measurements were taken at $1 \mathrm{kHz}$. Table III tabulates the result.

We measured frequency response of a sensing element in a much less elaborate facility than the Helmholtz coil. A simple exciting coil, constructed by hand-winding wire on a Poly-Vinyl-Chloride (PVC) tube of a $11.5 \mathrm{~cm}$ diameter, was driven by an oscillator/amplifier combination. The coil current was monitored. A 'first batch' probe (KY3A2F-1N0000) was placed inside the exciting coil with its axis approximately aligned with that of the low frequency Z-axis coil. The sensing element was connected to a digital oscilloscope with a $1 \mathrm{~m}$ long RG-58 coaxial cable with a BNC connector on one end and a pair of alligator clips on the other. The field was not uniform over the probe. But we believe this simple arrangement is sufficient to determine the relative sensitivity of a sensing element.

The measured result appears in Fig. 14. The sensing element response is normalized by the measured exciting coil current at each frequency. The response plotted along the ordinate is relative to its values at low frequencies. The abscissa is the frequency in a logarithmic scale.

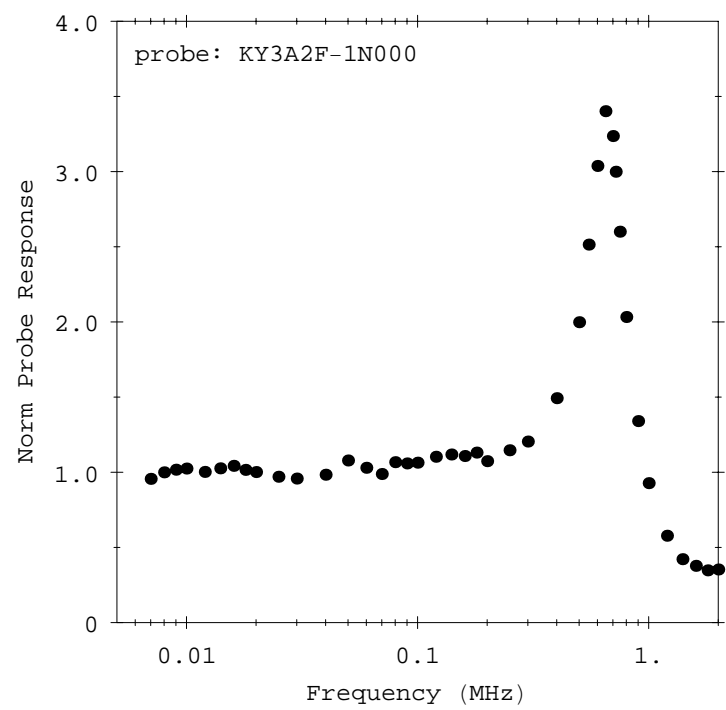

Fig. 14 Frequency response of a low-frequency Z-axis coil of an AT probe. The probe response is relative to its values at low frequencies.

The response increases gradually by about $25 \%$ as the frequency rises to a 'demarcation' point at about $300 \mathrm{kHz}$. The response then increases sharply to a large 'resonance-like' peak centered about $650 \mathrm{kHz}$. The response falls below unity just above $900 \mathrm{kHz}$, but is still about 0.2 even at 
$2 \mathrm{MHz}$. The signal phase has not been recorded in this measurement. But we believe that the phase is nearly linear with the frequency up to the 'demarcation' point. Under this assumption an upper limit of a usable frequency range is $300 \mathrm{kHz}$ when the phase is important. The upper limit is over $2 \mathrm{MHz}$ for the purpose of simply detecting the presence of a signal.

We have not yet completed frequency response measurement of high frequency coils. Their characterization to date is only in terms of the measured inductance, which is tabulated in Table III.

\section{ACKNOWLEGEMENT}

The authors are grateful for the support and encouragement received from Profs. Atuso Iiyoshi and Osamu Motojima of National Institute for Fusion Science (NIFS), Japan, and Prof. Robert Goldston and Dr. Kenneth Young of Princeton Plasma Physics Laboratory, U.S.A.

One of the authors (H.T.) would like to express his special gratitude to Prof. Iiyoshi for providing him with the initial impetus, continuing support, and funding for hardware to construct proto-types of his magnetic probe design. He began his work in late 1996 in collaboration with his NIFS colleagues, when Prof. Iiyoshi, then Director General of NIFS, invited the author as a short-term foreign visiting professor of the Institute. He continued his work after his return to Princeton as well as during his subsequent short-term visits to NIFS through the US/Japan collaboration under the Fusion Physics Planning Committee.

This work was supported in part by U.S. D.o.E. contract DE-AC02-76-CH03073. 


\section{References}

${ }^{1}$ I.H. Hutchinson, Principles of Plasma Diagnostics (Cambridge University Press, Cambridge, 1990).

${ }^{2}$ S.S. Medley, Rev. Sci. Instrum., 66, 297 (1995).

${ }^{3}$ J. Coonrod, M.G. Bell, R.J. Hawryluk, D. Mueller, and G.D. Tait, Rev. Sci. Instrum., 56, 941 (1985).

${ }^{4}$ J.L. Luxon and L.G. Davis, Fusion Technology, 8, 441 (1985).

${ }^{5}$ R.S. Granetz, I.H. Hutchinson, J. Gerolamo, W. Pina, and C. Tsui, Rev. Sci. Instrum., 61, 2967 (1990).

${ }^{6}$ E.J. Strait, Rev. Sci. Instrum., 67, 2538 (1996).

${ }^{7}$ A. Iiyoshi, et al, Nucl. Fusion 39, 1245 (1999).

${ }^{8}$ E.D. Fredrickson, K. McGuire, A. Cavallo, B. Grek, K.-I. Hattori, D. Johnson, and A.W. Morris, Rev. Sci. Instrum. 59, 1797 (1988).

${ }^{9}$ R.J. Hawryluk, et al., Phys. Plasmas 5, 1577 (1998).

${ }^{10}$ S. Wolfram, The Mathematica Book, 4th ed. (Wolfram Media/Cambridge Univ. Press, Champaign, IL/Cambridge, 1999).

${ }^{11}$ Y. Kubota and J. Kodaira, Rev. Sci. Instrum, 60, 958 (1989). 


\section{External Distribution}

Plasma Research Laboratory, Australian National University, Australia

Professor I.R. J ones, Flinders University, Australia

Professor J oão Canalle, Instituto de Fisica DEQ/IF - UERJ , Brazil

Mr. Gerson O. Ludwig, Instituto Nacional de Pesquisas, Brazil

Dr. P.H. Sakanaka, Instituto Fisica, Brazil

The Librarian, Culham Laboratory, England

Library, R61, Rutherford Appleton Laboratory, England

Mrs. S.A. Hutchinson, JET Library, England

Professor M.N. Bussac, Ecole Polytechnique, France

Librarian, Max-Planck-Institut für Plasmaphysik, Germany

J olan Moldvai, Reports Library, MTA KFKI-ATKI, Hungary

Dr. P. Kaw, Institute for Plasma Research, India

Ms. P.J . Pathak, Librarian, Insitute for Plasma Research, India

Ms. Clelia De Palo, Associazione EURATOM-ENEA, I taly

Dr. G. Grosso, Instituto di Fisica del Plasma, Italy

Librarian, Naka Fusion Research Establishment, J AERI, J apan

Library, Plasma Physics Laboratory, Kyoto University, J apan

Research Information Center, National Institute for Fusion Science, J apan

Dr. O. Mitarai, Kyushu Tokai University, J apan

Library, Academia Sinica, Institute of Plasma Physics, People's Republic of China

Shih-Tung Tsai, Institute of Physics, Chinese Academy of Sciences, People's Republic of China

Dr. S. Mirnov, TRINITI, Troitsk, Russian Federation, Russia

Dr. V.S. Strelkov, Kurchatov Institute, Russian Federation, Russia

Professor Peter Lukac, Katedra Fyziky Plazmy MFF UK, Mlynska dolina F-2, Komenskeho Univerzita, SK-842 15 Bratislava, Slovakia

Dr. G.S. Lee, Korea Basic Science Institute, South Korea

Mr. Dennis Bruggink, Fusion Library, University of Wisconsin, USA

Institute for Plasma Research, University of Maryland, USA

Librarian, Fusion Energy Division, Oak Ridge National Laboratory, USA

Librarian, Institute of Fusion Studies, University of Texas, USA

Librarian, Magnetic Fusion Program, Lawrence Livermore National Laboratory, USA

Library, General Atomics, USA

Plasma Physics Group, Fusion Energy Research Program, University of California at San Diego, USA

Plasma Physics Library, Columbia University, USA

Alkesh Punjabi, Center for Fusion Research and Training, Hampton University, USA

Dr. W.M. Stacey, Fusion Research Center, Georgia Institute of Technology, USA

Dr. J ohn Willis, U.S. Department of Energy, Office of Fusion Energy Sciences, USA

Mr. Paul H. Wright, Indianapolis, Indiana, USA 
The Princeton Plasma Physics Laboratory is operated by Princeton University under contract with the U.S. Department of Energy.

\author{
Information Services \\ Princeton Plasma Physics Laboratory \\ P.O. Box 451 \\ Princeton, NJ 08543
}

Phone: 609-243-2750

Fax: 609-243-2751

e-mail: pppl_info@pppl.gov

Internet Address: http://www.pppl.gov 